\title{
EXISTENCE AND NONEXISTENCE OF ENTIRE SOLUTIONS TO THE LOGISTIC DIFFERENTIAL EQUATION
}

\author{
MARIUS GHERGU AND VICENŢIU RĂDULESCU
}

Received 26 February 2003

We consider the one-dimensional logistic problem $\left(r^{\alpha} A\left(\left|u^{\prime}\right|\right) u^{\prime}\right)^{\prime}=r^{\alpha} p(r) f(u)$ on $(0, \infty), u(0)>0, u^{\prime}(0)=0$, where $\alpha$ is a positive constant and $A$ is a continuous function such that the mapping $t A(|t|)$ is increasing on $(0, \infty)$. The framework includes the case where $f$ and $p$ are continuous and positive on $(0, \infty)$, $f(0)=0$, and $f$ is nondecreasing. Our first purpose is to establish a general nonexistence result for this problem. Then we consider the case of solutions that blow up at infinity and we prove several existence and nonexistence results depending on the growth of $p$ and $A$. As a consequence, we deduce that the mean curvature inequality problem on the whole space does not have nonnegative solutions, excepting the trivial one.

\section{Introduction and the main results}

As pointed out in the recent monograph by Buttazzo et al. [3], one-dimensional variational problems deserve special attention. In fact, problems of this kind have their own characters. Sometimes, as we will also see in this paper, higherdimensional variational problems can be reduced to one-dimensional ones.

We study the following problem:

$$
\left[r^{\alpha} A\left(\left|u^{\prime}\right|\right) u^{\prime}\right]^{\prime}=r^{\alpha} p(r) f(u), \quad r>0, u(0)>0, u^{\prime}(0)=0,
$$

where $\alpha>0$, and $A$ satisfies

(A1) $A \in C[0, \infty), A(t)>0$ for $t>0$, the mapping $t A(|t|)$ is of class $C(\mathbb{R}) \cap$ $C^{1}(0, \infty)$ and $[t A(t)]^{\prime}>0$ for $t>0$.

We assume throughout the paper that $f$ is a continuous and nondecreasing function on $[0, \infty)$ satisfying $f(0)=0$, and $f>0$ on $(0, \infty)$. 
We suppose that $p$ is a positive continuous function on $[0, \infty)$ such that the mapping $g(r)=r^{-\alpha} \int_{0}^{r} s^{\alpha} p(s) d s$ satisfies either

(g1) $g(r) \rightarrow \infty$ as $r \rightarrow \infty$ or

(g2) $g^{\prime}(r) \geq M>0$ for all $r>0$.

Obviously, (g2) implies (g1).

Remark 1.1. (i) For $\alpha=N-1$ and $A(t)=t^{m-2}, m>1$, (1.1) becomes the $m$ Laplacian equation

$$
\operatorname{div}\left(|\nabla u|^{m-2} \nabla u\right)=p(r) f(u)
$$

in the radial case.

(ii) For $\alpha=N-1$ and $A(t)=\left(1+t^{2}\right)^{-1 / 2},(1.1)$ becomes the radial mean curvature equation, that is,

$$
\operatorname{div}\left(\frac{\nabla u}{\sqrt{1+|\nabla u|^{2}}}\right)=p(r) f(u)
$$

(iii) Typical functions $A$ that verify (A1):

$$
\begin{aligned}
& A(t)=t^{m-2}, \quad m>1, \\
& A(t)=\left(1+t^{2}\right)^{-\alpha}, \quad \alpha \leq \frac{1}{2}, \\
& A(t)=t^{2 m-2}\left(1+t^{2 m}\right)^{-1 / 2}, \quad m \geq 1 .
\end{aligned}
$$

(iv) Examples of functions $p$ satisfying (g1): $p(r)=r^{a}\left(1+r^{2}\right)^{b}$, provided $a+$ $2 b>-1 ; p(r)=e^{r} ; p(r)=\ln (2+r)$. The condition (g2) is fulfilled for $p(r)=r^{\gamma}$, $(\gamma>0) ; p(r)=e^{r}$.

Our first result concerns the nonexistence of the solution to problem (1.1) in the case where $\lim _{t \rightarrow \infty} t A(t)<\infty$.

Theorem 1.2. Assume that $A$ satisfies (A1), $\lim _{t \rightarrow \infty} t A(t)<\infty$, and p satisfies ( $\left.g 1\right)$. Then (1.1) has no positive solution.

Next we consider the case where

(A2) $\lim _{t \rightarrow \infty} A(t) / t^{m-2}=A_{0} \in(0, \infty)$, for some $m>1$.

We point out that the case $A(t)=t^{m-2}, m>2$, was studied in [11].

Remark 1.3. (i) Since $m>1$, (A2) leads to $\lim _{t \rightarrow \infty} t A(t)=+\infty$.

(ii) Functions satisfying both conditions (A1) and (A2) are given, for example, by (1.4) and (1.6), with $m>1$.

Define $\Psi:[0, \infty) \rightarrow[0, \infty)$ by $\Psi(t)=t^{2} A(t)-\int_{0}^{t} s A(s) d s, t>0$. From (A1), it follows that $\Psi$ is a continuous increasing function with $\Psi(0)=0$. 
From (A2), and l'Hospital's rule, we deduce that

$$
\lim _{t \rightarrow \infty} \frac{\Psi(t)}{t^{m}}=\frac{m-1}{m} A_{0} \in(0, \infty) .
$$

Theorem 1.4. Assume (A1), (A2), and (g2) hold. If

$$
\int^{\infty}\left(\int^{t} f(s) d s\right)^{-1 / m} d t<\infty,
$$

then (1.1) has no positive solutions.

In fact, the proof of Theorem 1.4 establishes that if problem (1.1) would have a solution then, necessarily, this solution blows up at infinity, that is, $u(r) \rightarrow+\infty$ as $r \rightarrow \infty$. Such a solution is called explosive or large. There is a great interest in the last few decades regarding the study of solutions that blow up at the boundary or at infinity. If $m=2$, then condition (1.8) is known as the Keller-Osserman condition (see $[6,13])$ and it plays a basic role in the treatment of elliptic equation that admits large solutions. Basic results in the study of large solutions for stationary problems have been recently obtained in $[1,2,4,5,7,8,9,10,11]$.

Theorem 1.5. Assume (A1), (A2), and (g1) hold. If

$$
\int^{\infty}\left(\int^{t} f(s) d s\right)^{-1 / m} d t=\infty
$$

then (1.1) has at least one positive solution. Moreover, this solution is large.

Our next result gives an estimate of the growth of a solution of (1.1) in case if $f$ is bounded. More precisely, we prove the following theorem.

Theorem 1.6. Assume (A1), (A2), and ( $g 1)$ hold and $f$ is bounded. If $u$ is a positive solution of (1.1), then

$$
\lim _{r \rightarrow \infty} \frac{u(r)}{\int_{0}^{r} g^{1 /(m-1)}(s) d s}=\lim _{r \rightarrow \infty}\left(\frac{1}{A_{0}} f(r)\right)^{1 /(m-1)} .
$$

\section{Proofs}

If $u$ is a positive solution of (1.1), then

$$
\begin{array}{cc}
{\left[A\left(\left|u^{\prime}\right|\right) u^{\prime}\right]^{\prime}+\frac{\alpha}{r} A\left(\left|u^{\prime}\right|\right) u^{\prime}=p(r) f(r) \quad \forall r>0,} \\
A\left(\left|u^{\prime}(r)\right|\right) u^{\prime}(r)=r^{-\alpha} \int_{0}^{r} s^{\alpha} p(s) f(u(s)) d s \quad \forall r>0 .
\end{array}
$$

We deduce that $A\left(\left|u^{\prime}(r)\right|\right) u^{\prime}(r)>0$ for $r>0$ which implies $u^{\prime}(r)>0$. Since $f$ is nondecreasing, it follows that

$$
A\left(u^{\prime}(r)\right) u^{\prime}(r) \leq g(r) f(u(r)) \quad \forall r>0 .
$$


Now (2.1) and (2.3) yield

$$
\left[A\left(u^{\prime}\right) u^{\prime}\right]^{\prime}(r) \geq\left(p(r)-\alpha \frac{g(r)}{r}\right) f(u(r)) \quad \forall r>0,
$$

that is,

$$
\left[A\left(u^{\prime}\right) u^{\prime}\right]^{\prime}(r) \geq g^{\prime}(r) f(u(r)) \quad \forall r>0 .
$$

Proof of Theorem 1.2. Arguing by contradiction, let $u$ be a solution of (1.1). Since $u(0)>0$ and $f, u$ are nondecreasing functions, from (2.1) we get

$$
A\left(u^{\prime}(r)\right) u^{\prime}(r) \geq g(r) f(u(0)) \quad \forall r>0 .
$$

On the other hand, $\lim _{t \rightarrow \infty} t A(t)<\infty$, which implies that $A\left(u^{\prime}(r)\right) u^{\prime}(r)$ is bounded on $[0, \infty)$. This fact and the above inequality lead to a contradiction since $g(r) \rightarrow \infty$ as $r \rightarrow \infty$ and $f(u(0))>0$. The proof of Theorem 1.2 is now complete.

Proof of Theorem 1.4. Assume by contradiction that problem (1.1) has a positive solution $u$. From (2.6), we get $u^{\prime}(r) \rightarrow \infty$ as $r \rightarrow \infty$ and so, by Remark 1.3, $u(r) \rightarrow$ $\infty$ as $r \rightarrow \infty$.

By (g2) and (2.5), we have

$$
\left[A\left(u^{\prime}\right) u^{\prime}\right]^{\prime}(s) \geq M f(u(s)) \quad \forall s>0 .
$$

Multiplying by $u^{\prime}$ the above inequality and integrating on $[0, r]$, we obtain

$$
\int_{0}^{r}\left[A\left(u^{\prime}\right) u^{\prime}\right]^{\prime}(s) u^{\prime}(s) d s \geq M \int_{0}^{r} f(u(s)) u^{\prime}(s) d s \quad \forall r>0 .
$$

Now, integration by parts yields

$$
A\left(u^{\prime}(r)\right)\left(u^{\prime}(r)\right)^{2}-\int_{0}^{r} A\left(u^{\prime}(s)\right) u^{\prime}(s) u^{\prime \prime}(s) d s \geq M \int_{0}^{r} f(u(s)) u^{\prime}(s) d s
$$

for all $r>0$. By a change of variables, we now find

$$
A\left(u^{\prime}(r)\right)\left(u^{\prime}(r)\right)^{2}-\int_{0}^{u^{\prime}(r)} t A(t) d t \geq M \int_{u(0)}^{u(r)} f(t) d t \quad \forall r>0 .
$$

Hence,

$$
\Psi\left(u^{\prime}(r)\right) \geq M \int_{u(0)}^{u(r)} f(u(s)) d s \quad \forall r>0 .
$$

By (1.7) and using the fact that $u^{\prime}(r) \rightarrow+\infty$ as $r \rightarrow \infty$, there exist $r_{0}>0$ and a positive constant $C>0$ such that

$$
u^{\prime}(r) \geq C\left(\int_{u(0)}^{u(r)} f(u(s)) d s\right)^{1 / m} \quad \forall r>r_{0}
$$


Hence,

$$
\left(\int_{u(0)}^{u(r)} f(u(s)) d s\right)^{-1 / m} u^{\prime}(r) \geq C \quad \forall r>r_{0} .
$$

Integrating this inequality on $\left[r_{0}, r\right]$, we find

$$
\int_{u\left(r_{0}\right)}^{u(r)}\left(\int_{u(0)}^{t} f(u(s)) d s\right)^{-1 / m} d t \geq C\left(r-r_{0}\right) \quad \forall r>r_{0} .
$$

Letting $r \rightarrow \infty$ in the above relation, we get

$$
\int_{u\left(r_{0}\right)}^{\infty}\left(\int_{u(0)}^{t} f(u(s)) d s\right)^{-1 / m} d t=+\infty
$$

This contradicts our assumption (1.8) and completes the proof.

Proof of Theorem 1.5. The existence of a solution $u$ of (1.1) in a certain interval $[0, R)$ follows by the classical arguments of ODEs. Assume, by contradiction, that the maximal interval of existence of $u$ is a finite interval $[0, R), R<\infty$.

We first claim that $u(R-0)=\lim _{r>R} u(r)=\infty$. Indeed, since $u^{\prime} \geq 0$ on $[0, R)$, it follows that $u(R-0)$ exists in $(0, \infty)$. From $(2.1)$, we deduce that $u^{\prime}(R-0)$ exists and is finite. Then, by standard arguments for initial value problems, it follows that $u$ can be extended as a solution on an interval $[0, R+\varepsilon), \varepsilon>0$, which contradicts the maximality of $R$. Hence, $u(R-0)=\infty$.

Using (2.1) and the fact that $A\left(u^{\prime}\right) u^{\prime} \geq 0$ on $[0, R)$, we have

$$
\left[A\left(u^{\prime}\right) u^{\prime}\right]^{\prime}(s) \leq g(s) f(u(s)) \leq C_{0} f(u(s)) \quad \forall 0<s<R,
$$

where $C_{0}=\max _{r \in[0, R]} g(r)>0$. Multiplying the above inequality by $u^{\prime} \geq 0$ and integrating on $[0, r]$, we have

$$
\Psi\left(u^{\prime}(r)\right) \leq C_{0} \int_{u(0)}^{u(r)} f(u(s)) d s \quad \forall 0<r<R .
$$

According to (1.7), there exists $R_{0} \in(0, R)$ such that

$$
u^{\prime}(r) \leq C_{1}\left(\int_{u(0)}^{u(r)} f(s) d s\right)^{1 / m} \quad \forall r \in\left(R_{0}, R\right),
$$

where $C_{1}>0$ is a constant independent of $f$ and $u$. Hence,

$$
\left(\int_{u(0)}^{u(r)} f(s) d s\right)^{-1 / m} u^{\prime}(r) \leq C_{1} \quad \forall r \in\left(R_{0}, R\right) .
$$


An integration over $\left[R_{0}, r\right], r<R$, and a change of variable lead to

$$
\int_{u\left(R_{0}\right)}^{u(r)}\left(\int_{u(0)}^{t} f(u(s)) d s\right)^{-1 / m} d t \leq C_{1}\left(r-R_{0}\right) \quad \forall r>r_{0} .
$$

Now, letting $r \succ R$, we find

$$
\int_{u\left(R_{0}\right)}^{\infty}\left(\int_{u(0)}^{t} f(u(s)) d s\right)^{-1 / m} d t \leq C_{1}\left(R-R_{0}\right)<\infty,
$$

which contradicts our assumption (1.9). We conclude that there exists a solution of (1.1) and the proof is now complete.

Proof of Theorem 1.6. Applying l'Hospital's rule, we have

$$
\lim _{r \rightarrow \infty} \frac{u(r)}{\int_{0}^{r} g^{1 /(m-1)}(s) d s}=\lim _{r \rightarrow \infty} \frac{u^{\prime}(r)}{g^{1 /(m-1)}(r)} .
$$

From (A2), we get

$$
\lim _{r \rightarrow \infty} \frac{u^{\prime m-1}(r)}{g(r)}=\lim _{r \rightarrow \infty} \frac{u^{\prime m-1}(r)}{A\left(u^{\prime}(r)\right) u^{\prime}(r)} \frac{A\left(u^{\prime}(r)\right) u^{\prime}(r)}{g(r)}=\frac{1}{A_{0}} \lim _{r \rightarrow \infty} \frac{A\left(u^{\prime}(r)\right) u^{\prime}(r)}{g(r)} .
$$

By (2.1), l'Hospital's rule, and the fact that $u(r) \rightarrow+\infty$ as $r \rightarrow \infty$, we deduce that

$$
\lim _{r \rightarrow \infty} \frac{A\left(u^{\prime}(r)\right) u^{\prime}(r)}{g(r)}=\lim _{r \rightarrow \infty} \frac{\int_{0}^{r} s^{\alpha} p(s) f(u(s)) d s}{\int_{0}^{r} s^{\alpha} p(s) d s}=\lim _{r \rightarrow \infty} f(u(r))=\lim _{r \rightarrow \infty} f(r) .
$$

Now, (2.22), (2.23), and (2.24) lead to

$$
\lim _{r \rightarrow \infty} \frac{u(r)}{\int_{0}^{r} g^{1 /(m-1)}(s) d s}=\lim _{r \rightarrow \infty}\left(\frac{1}{A_{0}} f(r)\right)^{1 /(m-1)} .
$$

This completes our proof.

\section{Applications to the $N$-dimensional case}

In this section, we show how the previous results can be applied to the corresponding problem (1.1) in the $N$-dimensional case, that is,

$$
\operatorname{div}[A(|\nabla u|) \nabla u] \geq p(|x|) f(u), \quad x \in \mathbb{R}^{N} .
$$

Many papers have been devoted to the semilinear case $A \equiv 1$ (see, e.g., [1, 2, $4,5,6,7,8,9,10,11,13$ ] and the references therein). In [12], Naito and Usami studied the case $p \equiv 1$ and $A$ satisfying (A1).

A very useful tool is the following comparison principle. 
Proposition 3.1. Assume that (A1) holds. Let $\Omega$ be a bounded domain in $\mathbb{R}^{N}$ with smooth boundary. Let $u$ be a nonnegative entire solution of (3.1) and let $v \in$ $C(\bar{\Omega}) \cap C^{1}(\Omega)$ be a positive function satisfying

$$
\begin{gathered}
A(|\nabla v|) \nabla v \in C^{1}(\Omega), \\
\operatorname{div}[A(|\nabla v|) \nabla v] \leq p(|x|) f(v) \quad \text { in } \Omega, \\
u \leq v \quad \text { on } \partial \Omega .
\end{gathered}
$$

Then $u \leq v$ in $\Omega$.

We also have the following proposition.

Proposition 3.2. Assume that (A1) holds. If inequality (3.1) has a nonnegative entire solution $u \neq 0$, then there exists a positive solution $v$ of (1.1) corresponding to $\alpha=N-1$.

The proofs of Propositions 3.1 and 3.2 are similar to those for the case $p \equiv 1$, which can be found in [12].

Now we state the results that can be reduced to the one-dimensional case of the inequality problem (3.1).

Theorem 3.3. Assume that A satisfies (A1), $\lim _{t \rightarrow \infty} t A(t)<+\infty$, and $p$ satisfies ( $g 1)$. If $u$ is a nonnegative entire solution of (3.1), then $u \equiv 0$.

Proof. Arguing by contradiction, assume that $u \neq 0$. Then, by Proposition 3.2, problem (1.1) has a positive solution for $\alpha=N-1$, which contradicts Theorem 1.2. Hence, $u \equiv 0$.

Theorem 3.4. Assume that (A1), (A2), and (g2) hold. If

$$
\int^{\infty}\left(\int^{t} f(s) d s\right)^{-1 / m} d t<\infty
$$

and $u$ is a nonnegative entire solution of (3.1), then $u \equiv 0$.

Proof. By contradiction, assume that $u \neq 0$. Then, by Proposition 3.2, (1.1) has a positive solution for $\alpha=N-1$, which contradicts Theorem 1.4. Hence, $u \equiv 0$.

Theorem 3.5. Assume (A1), (A2), and (g1) hold. If

$$
\int^{\infty}\left(\int^{t} f(s) d s\right)^{-1 / m} d t=\infty,
$$

then inequality (3.1) has infinitely many positive entire large solutions.

Proof. By Theorem 1.5, for any $a>0$, there exists a positive solution $v_{a}$ of (1.1) with $v_{a}(0)=a$. Furthermore, we have $v_{a}(r) \rightarrow+\infty$ as $r \rightarrow \infty$. Thus, $u_{a}(x):=$ $v_{a}(|x|), x \in \mathbb{R}^{N}$, is a positive entire large solution of (3.1). 
Corollary 3.6. Assume that (A1), (A2), and (g2) hold. Then (3.1) has a positive entire (large) solution if and only if

$$
\int^{\infty}\left(\int^{t} f(s) d s\right)^{-1 / m} d t=+\infty
$$

Examples. (i) If $p$ satisfies ( $\mathrm{g} 1$ ) and $u$ is a nonnegative entire solution of the mean curvature inequality problem

$$
\operatorname{div}\left(\frac{\nabla u}{\sqrt{1+|\nabla u|^{2}}}\right) \geq p(|x|) f(u), \quad x \in \mathbb{R}^{N}
$$

then $u \equiv 0$.

(ii) If $p$ satisfies (g2), then the inequality

$$
\operatorname{div}\left(\frac{\nabla u}{\left(1+|\nabla u|^{2}\right)^{\alpha}}\right) \geq p(|x|) e^{u}, \quad x \in \mathbb{R}^{N}, \alpha<\frac{1}{2},
$$

has no positive entire solutions, while the inequality

$$
\operatorname{div}\left(\frac{\nabla u}{\left(1+|\nabla u|^{2}\right)^{\alpha}}\right) \geq p(|x|) u^{\gamma}, \quad x \in \mathbb{R}^{N}, \alpha<\frac{1}{2}, \gamma \geq 0,
$$

has positive entire (large) solutions if and only if $\gamma \leq 1-2 \alpha$.

(iii) If $p$ satisfies (g2), then the inequality

$$
\operatorname{div}\left(|\nabla u|^{m-2} \nabla u\right) \geq p(|x|) u^{\gamma}, \quad x \in \mathbb{R}^{N}, m>1, \gamma \geq 0 .
$$

has positive entire (large) solutions if and only if $\gamma \leq m-1$.

Remark 3.7. The assumptions $(g 1)$ and $(g 2)$ are sufficient but not necessary for the existence of a solution to inequality (3.1). As a counterexample we can take $A \equiv 1, f(r)=r, p(r)=2 N /\left(r^{2}+1\right)$. It is obvious that (g1) and (g2) fail to hold but the inequality problem (3.1) has the positive entire large solution $u(x)=$ $|x|^{2}+1$.

\section{Acknowledgment}

This work was partially supported by a research grant through the Romanian Academy of Sciences.

\section{References}

[1] C. Bandle, A. Greco, and G. Porru, Large solutions of quasilinear elliptic equations: existence and qualitative properties, Boll. Un. Mat. Ital. B (7) 11 (1997), no. 1, 227-252.

[2] C. Bandle and M. Marcus, "Large" solutions of semilinear elliptic equations: existence, uniqueness and asymptotic behaviour, J. Anal. Math. 58 (1992), 9-24. 
[3] G. Buttazzo, M. Giaquinta, and S. Hildebrandt, One-Dimensional Variational Problems, Oxford Lecture Series in Mathematics and Its Applications, vol. 15, The Clarendon Press, Oxford University Press, New York, 1998.

[4] F. Şt. Cîrstea and V. D. Rădulescu, Blow-up boundary solutions of semilinear elliptic problems, Nonlinear Anal., Ser. A: Theory Methods 48 (2002), no. 4, 521-534.

[5] Y. Du and Q. Huang, Blow-up solutions for a class of semilinear elliptic and parabolic equations, SIAM J. Math. Anal. 31 (1999), no. 1, 1-18.

[6] J. B. Keller, On solutions of $\Delta u=f(u)$, Comm. Pure Appl. Math. 10 (1957), 503-510.

[7] A. C. Lazer and P. J. McKenna, Asymptotic behavior of solutions of boundary blow-up problems, Differential Integral Equations 7 (1994), no. 3-4, 1001-1019.

[8] C. Loewner and L. Nirenberg, Partial differential equations invariant under conformal or projective transformations, Contributions to Analysis: A Collection of Papers Dedicated to Lipman Bers (L. V. Ahlfors, I. Kra, B. Maskit, and L. Nirenberg, eds.), Academic Press, New York, 1974, pp. 245-272.

[9] M. Marcus, On solutions with blow-up at the boundary for a class of semilinear elliptic equations, Developments in Partial Differential Equations and Applications to Mathematical Physics (Ferrara, 1991) (G. Buttazzo, G. P. Galdi, and L. Zanghirati, eds.), Plenum Press, New York, 1992, pp. 65-77.

[10] M. Marcus and L. Véron, Uniqueness and asymptotic behavior of solutions with boundary blow-up for a class of nonlinear elliptic equations, Ann. Inst. H. Poincaré Anal. Non Linéaire 14 (1997), no. 2, 237-274.

[11] A. Mohammed, G. Porcu, and G. Porru, Large solutions to some non-linear O.D.E. with singular coefficients, Nonlinear Anal. 47 (2001), 513-524.

[12] Y. Naito and H. Usami, Entire solutions of the inequality $\operatorname{div}(A(|D u|) D u) \geq f(u)$, Math. Z. 225 (1997), no. 1, 167-175.

[13] R. Osserman, On the inequality $\Delta u \geq f(u)$, Pacific J. Math. 7 (1957), 1641-1647.

Marius Ghergu: Department of Mathematics, University of Craiova, 200585 Craiova, Romania

E-mail address: ghergu@inf.ucv.ro

Vicenţiu Rădulescu: Department of Mathematics, University of Craiova, 200585 Craiova, Romania

E-mail address: radulescu@inf.ucv.ro 


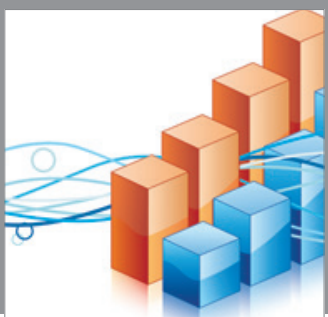

Advances in

Operations Research

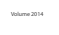

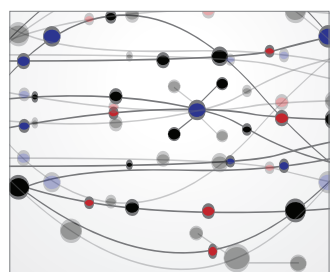

\section{The Scientific} World Journal
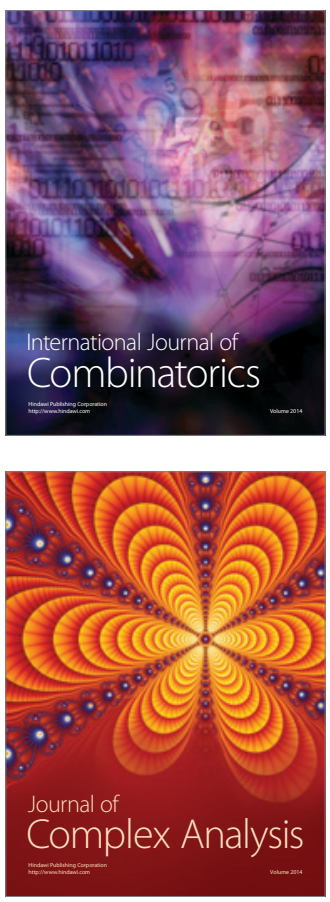

International Journal of

Mathematics and

Mathematical

Sciences
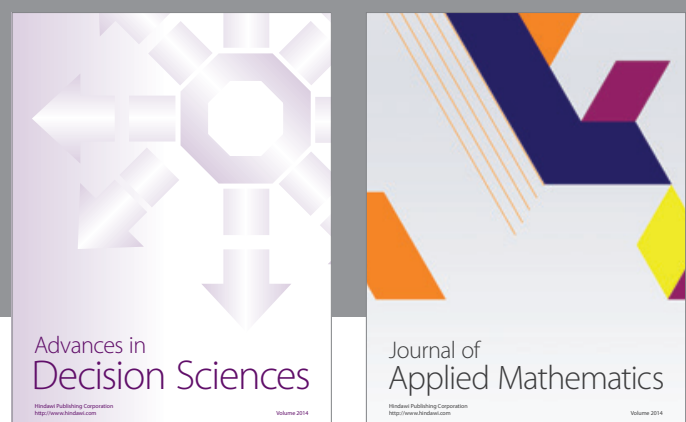

Journal of

Applied Mathematics
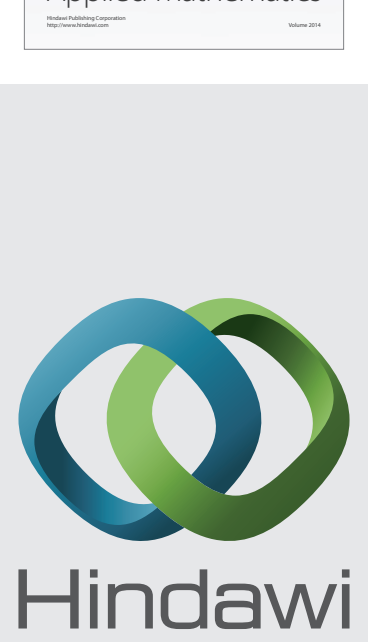

Submit your manuscripts at http://www.hindawi.com
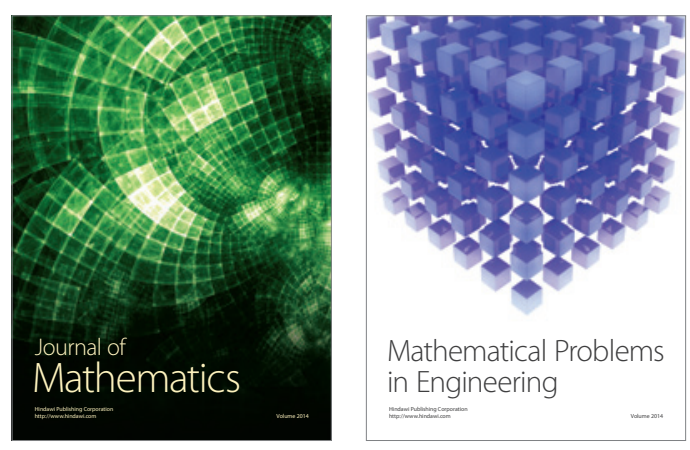

Mathematical Problems in Engineering
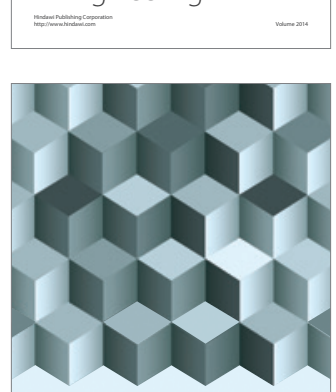

Journal of

Function Spaces
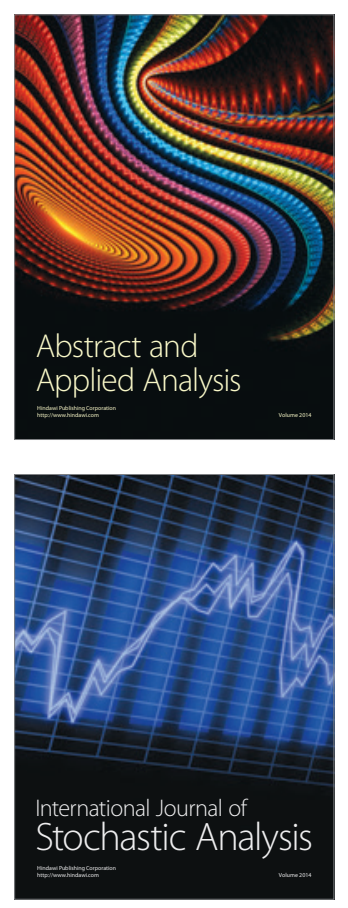

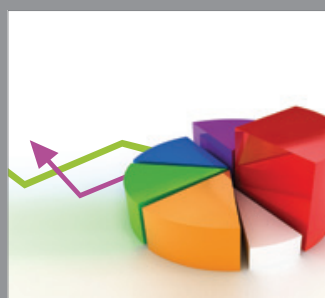

ournal of

Probability and Statistics

Promensencen
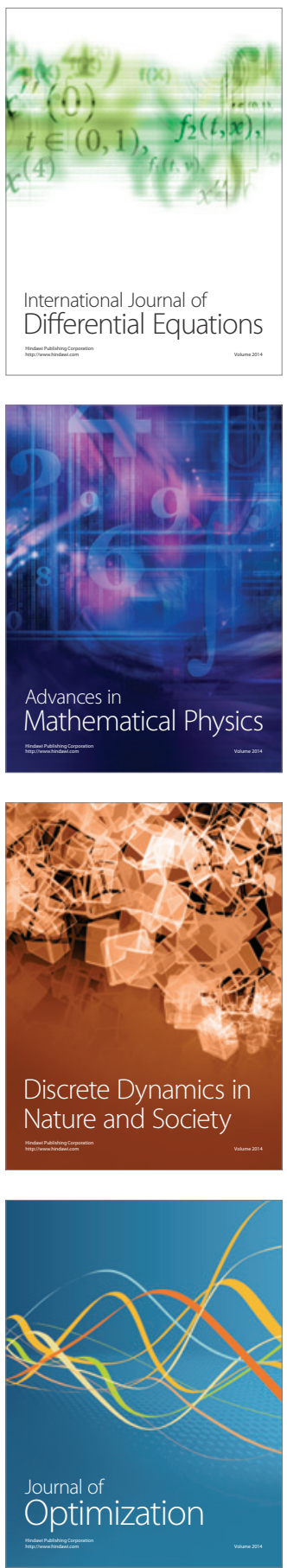